\title{
Quelques mots sur mon association
}

\author{
par Zack Dumont
}

Les autres agents présidentiels et directrices générales, Jody Ciufo, et moi avons entamé un dialogue avec nos membres sous forme de forums de discussion. Nous avons reçu des commentaires constructifs, abordé des préoccupations, répondu à des questions et nous en avons aussi posé. Parmi celles-ci : «Que signifie pour vous la Société canadienne des pharmaciens d'hôpitaux (SCPH)? ». Pour moi, la réponse est simple : la SCPH est et sera toujours ma communauté. Bien que la diversité de pensée soit importante à mes yeux, j'ai été heureux de constater que beaucoup partageaient ce point de vue, puisque le mot « communauté » est celui qui est ressorti le plus souvent.

J'admire cette communauté depuis plus de 12 ans, lorsque j'étais encore étudiant. Lors des réunions et des activités de la Société, je rêvais à la manière dont la SCPH avait vu le jour. J'imaginais, qu'autrefois, il y a plus de 70 ans, lorsque les leaders de la profession se réunissaient, ils disaient peut-être : "L'union fait la force. Et si nous nous rencontrions régulièrement? Et si nous nous inspirions un peu de ce que font nos collègues pour accomplir certaines tâches sur notre lieu de travail? Et si nous nous réunissions à nouveau pour mettre en commun nos résultats? » Ces interrogations sont certainement très réductrices, mais quelle que soit la manière dont tout cela s'est vraiment passé, j'aime la vitalité de ces novateurs qui ont su voir la possibilité de créer quelque chose - comme une coopérative quand personne n'y avait pensé, quand personne ne prêtait attention à eux. Qu'est-ce qui les a poussés à agir ainsi? Ces dirigeants étaient non seulement animés d'une vision, mais ils avaient aussi les compétences pour la concrétiser.

Aujourd'hui, la SCPH est plus forte que jamais. Il me semble que ses membres ressentent cette énergie. La SCPH demeure votre Société, votre coopérative. Quand je dis " votre ", je veux vraiment dire qu'elle est totalement vôtre. Ma responsabilité consiste simplement à la diriger au cours des prochaines années, mais elle ne cessera jamais d'être entre vos mains. Une autre personne me succèdera le moment venu. Entretemps, la Société reste votre lieu de rencontre. Notre communauté ne s'arrêtera jamais, dans la mesure où nous voulons qu'il en soit ainsi.

Qu'est-ce qui nous unit? Quel dénominateur commun nous donne l'impression d'être chez nous? Notre récent sondage nous éclaire. Les membres nous disent qu'ils sont très satisfaits de nos offres pédagogiques, de ce journal et de nos programmes de résidence. Pour moi, ces moyens font partie de nos mécanismes principaux de communication, de défense et de promotion de l'une de nos valeurs communes : œuvrer pour les patients, avec comme unique objectif l'amélioration de leur condition. Toutes les décisions de pharmacothérapie que nous prenons ou que nous soutenons, que ce soit de commencer, d'arrêter ou de modifier une thérapie, visent à améliorer la situation des patients.

Je me sens intégré dans la Société, car je peux y voir des leaders et des modèles qui remportent des prix, qui obtiennent des bourses et aussi parce que je me suis mêlé à eux lors des activités de la Société. La SCPH est un endroit incontournable. Elle m’a façonné; elle m'inspire de la fierté et je lui en suis reconnaissant. Au cours des prochaines années, je prévois démontrer ma gratitude en étoffant et en renforçant notre communauté : une communauté de diversité et d'inclusion. Je prévois aussi de me concentrer sur l'amélioration des résultats pour les patients.

Il ne s'agit là que de mon plan. Un leadership formel n'est peut-être pas fait pour vous. Je vous encourage cependant à vous engager dans votre association professionnelle. Une Société comme la SCPH est en effet plus grande que la somme de ses parties, et en tant que membre engagé, vous obtiendrez d'elle plus que vous ne pourrez lui offrir. Votre association professionnelle : une communauté qui mérite votre dévouement.

[Traduction par l'éditeur]

Zack Dumont, BSP, ACPR, M. S. (Pharm.), est président et agent de liaison interne de la Société canadienne des pharmaciens d'hôpitaux. 